\title{
Block based Channel Estimation Algorithms for OFDM- IEEE 802.16e (Mobile WiMAX) System
}

\author{
Manwinder Singh \\ Faculty ECE Deptt \\ RIEIT Railmajra \\ Distt. SBS Nagar, Punjab
}

\author{
Maninder Singh \\ Faculty ECE Deptt \\ LLRIET, Moga \\ Distt Moga, Punjab
}

\author{
Anudeep Goraya \\ Faculty ECE Deptt \\ RIEIT Railmajra \\ Distt. SBS Nagar, Punjab
}

\begin{abstract}
The objective of this study is improving channel estimation accuracy in OFDM system because channel state information is required for signal detection at receiver and its accuracy affects the overall performance of system and it is essential to improve the channel estimation for more reliable communications. OFDM system was chosen in this study because it has been widely used today due to its high data rate, channel capacity and its adequate performance in frequency selective fading channels. The pilots were inserted among subcarriers in transmitter with distances emerged of sampling theory then Least Square (LS) method \& minimum mean-square error (MMSE), was chosen for initial channel estimation in pilots at receiver, using applicable proposed receiver, which has simple and usable structure, then channel state information was estimated by linear interpolator in information subcarriers, which uses two adjacent channel estimation in pilots to compute channel in another subcarriers and an LMS iterative algorithm, including a feedback of output is added to system. This algorithm uses the channel estimation of last iteration in current estimation. Adding a LMS iterative algorithm to system, improves the channel estimation performance. Simulation results proved the acceptable BER performance of iterative channel estimation algorithm, which is closed to the ideal channel. The low complexity proposed receiver including LMS algorithm, has a higher efficiency than conventional methods(without channel estimation \& LMMSE ) and it can work in lower amount of SNRs.
\end{abstract}

\section{General Terms}

Least Square (LS), Minimum Mean-Square Error (MMSE). Algorithms et al.

\section{Keywords}

OFDM, AWGN, Rayleigh, LS Channel Estimation, LMMSE Channel Estimation.

\section{INTRODUCTION}

OFDM is becoming widely applied in wireless communications systems due to its high rate transmission capability with high bandwidth efficiency and its robustness with regard to multi-path fading and delay [1]. It has been used in digital audio broadcasting (DAB) systems, digital video broadcasting (DVB) systems, digital subscriber line (DSL) standards, and wireless LAN standards such as the American IEEE ${ }^{\circledR}$ Std. $802.11^{\mathrm{TM}}(\mathrm{WiFi})$ and its European equivalent HIPRLAN/2. It has also been proposed for wireless broadband access standards such as IEEE Std. $802.16^{\mathrm{TM}}$ (WiMAX) and as the core technique for the fourthgeneration $(4 \mathrm{G})$ wireless mobile communications. The use of differential phase-shift keying (DPSK) in OFDM systems avoids need to track a time varying channel; however, it limits the number of bits per symbol and results in a $3 \mathrm{~dB}$ loss in signal-to-noise ratio (SNR). Coherent modulation allows arbitrary signal constellations, but efficient channel estimation strategies are required for coherent detection and decoding.

There are two main problems in designing channel estimators for wireless OFDM systems. The first problem is the arrangement of pilot information, where pilot means the reference signal used by both transmitters and receivers. The second problem is the design of an estimator with both low complexity and good channel tracking ability. The two problems are interconnected. In general, the fading channel of OFDM systems can be viewed as a two-dimensional (2D) signal (time and frequency). Several previous works have investigated the performance of CEs assisted from various allocation patterns of the pilot/training symbols [4], [5]. Meanwhile, several prior channel estimators (CEs) [6]-[8] simultaneously exploited both time-directional and frequencydirectional correlations of the studied channel. The optimal channel estimator in terms of mean-square error is based on 2D Wiener filter interpolation. Unfortunately, such a 2D estimator structure is too complex for practical implementation. The combination of high data rates and low bit error rates in OFDM systems necessitates the use of estimators that have both low complexity and high accuracy, where the two constraints work against each other and a good trade-off is needed. The one-dimensional (1D) channel estimations are usually adopted in OFDM systems to accomplish the trade-off between complexity and accuracy [1-7]. The two basic 1D channel estimations are block-type pilot channel estimation and comb-type pilot channel estimation, in which the pilots are inserted in the frequency direction and in the time direction, respectively. The estimations for the block-type pilot arrangement can be based on least square (LS), minimum mean-square error (MMSE).

The rest of the paper is organized as follows: Section II Describe system description which is used in this paper. Section III describes the block based pilot channel estimation. In section IV, simulation \& results Discussion is described. Finally, conclusions \& Future work are presented in section V.

\section{SYSTEM DISCRIPTION}

The basic idea underlying OFDM systems is the division of the available frequency spectrum into several subcarriers. To obtain a high spectral efficiency, the frequency responses of the subcarriers are overlapping and orthogonal, hence the name OFDM. This orthogonality can be completely maintained with a small price in a loss in SNR, even though the signal passes through a time dispersive fading channel, by introducing a cyclic prefix (CP)[8-11]. A block diagram of a baseband OFDM system is shown in Figure 1. 
The binary information is first grouped, coded, and mapped according to the modulation in a "signal mapper." After the guard band is inserted, an N-point inverse discrete-time Fourier transform (IDFTN) block transforms the data sequence into time domain (note that $\mathrm{N}$ is typically 256 or larger). Following the IDFT block, a cyclic extension of time length $\mathrm{T}_{\mathrm{G}}$, chosen to be larger than the expected delay spread, is inserted to avoid inter-symbol and inter-carrier interferences[13]. The D/A converter contains low-pass filters with bandwidth $1 / T_{S}$, where $T_{S}$ is the sampling interval.

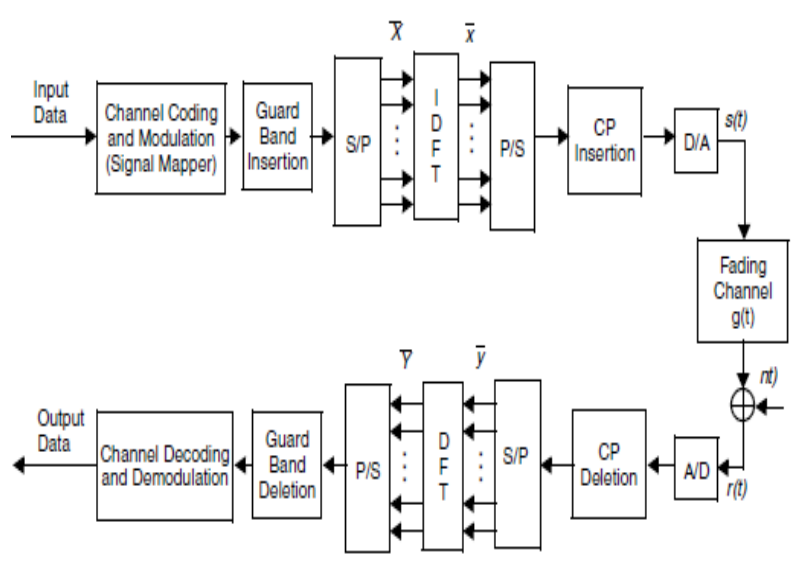

Fig 1: Block diagram of a baseband OFDM system

The channel is modeled as an impulse response $\mathrm{g}(\mathrm{t})$ followed by the complex additive white Gaussian noise (AWGN) n(t), where $\alpha_{\mathrm{m}}$ is a complex values and $0 \leq \tau_{\mathrm{m}} \mathrm{T}_{\mathrm{S}} \leq \mathrm{T}_{\mathrm{G}}$.

$$
g(t)=\sum_{m=1}^{M} \alpha_{m} \delta\left(t-\tau_{m} T_{S}\right)
$$

At the receiver, after passing through the analog-to-digital converter (ADC) and removing the CP, the DFTN is used to transform the data back to frequency domain. Lastly, the binary information data is obtained back after the demodulation and channel decoding.

Let $\bar{X}=\left[X_{k}\right]^{T}$ and $\bar{Y}=\left[Y_{k}\right]^{T}$ where $\mathrm{k}=0,1,2, \ldots \ldots .(\mathrm{N}-1)$ denote the input data of IDFT block at the transmitter and the output data of DFT block at the receiver, respectively. Let $\bar{g}=\left[g_{n}\right]^{T}$ and $\bar{n}=\left[n_{n}\right]^{T}$ where $\mathrm{n}=0,1,2, \ldots .(\mathrm{N}-1)$ denote the sampled channel impulse response and AWGN, respectively. Define the input matrix $\underline{X}=\operatorname{diag}(\bar{X})$ and the DFT-matrix,

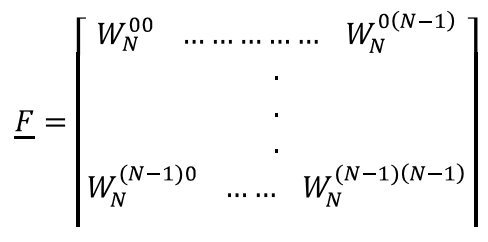

Where

$$
W_{N}^{i, k}=\left(\frac{1}{\sqrt{N}}\right)^{-j 2 \pi\left(\frac{i k}{N}\right)}
$$

Also define

$$
\bar{H}=\mathrm{DFT}_{\mathrm{N}}(\bar{g})=\underline{F} \bar{g} \& \bar{N}=\underline{F} \bar{n}
$$

Under the assumption that the interferences are completely eliminated [1-3], you can derive:

$$
\begin{gathered}
\bar{Y}=\operatorname{DFT}_{\mathrm{N}}\left(\operatorname{IDFT}_{\mathrm{N}}(\bar{X}) \otimes \bar{g}+\bar{n}\right) \\
=\underline{X F \bar{g}+\bar{N}} \\
=\underline{X} \bar{H}+\bar{N}
\end{gathered}
$$

This equation demonstrates that an OFDM system is equivalent to a transmission of data over a set of parallel channels.

As a result, the fading channel of the OFDM system can be viewed as a $2 \mathrm{D}$ lattice in a time-frequency plane, which is sampled at pilot positions and the channel characteristics between pilots are estimated by interpolation. The art in designing channel estimators is to solve this problem with a good trade-off between complexity and performance.

The two basic 1D channel estimations in OFDM systems are illustrated in Figure 2. The first one, block-type pilot channel estimation, is developed under the assumption of slow fading channel,

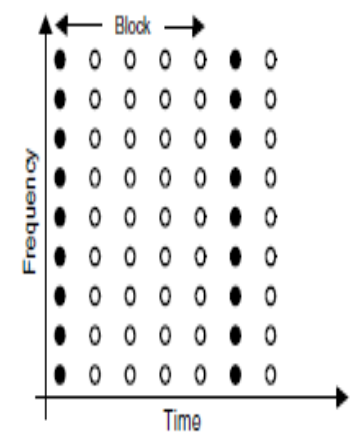

Block-type pilot channel estimation

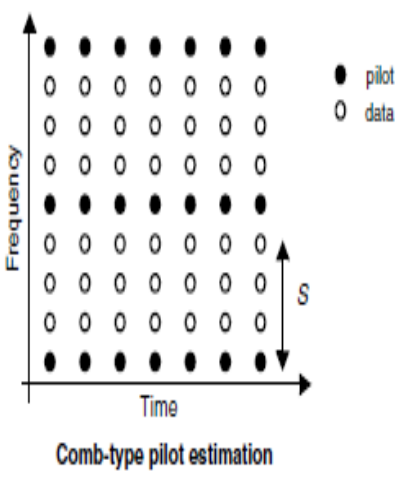

Fig 2: Two Basic Types of Pilot Arrangement for OFDM Channel Estimations

and it is performed by inserting pilot tones into all subcarriers of OFDM symbols within a specific period. The second one, comb-type pilot channel estimation, is introduced to satisfy the need for equalizing when the channel changes even from one OFDM block to the subsequent one. It is thus performed by inserting pilot tones into certain subcarriers of each OFDM symbol, where the interpolation is needed to estimate the conditions of data subcarriers. The strategies of these two basic types are analyzed in the next sections.

\section{BLOCK BASED PILOT CHANNEL ESTIMATION}

In block-type pilot-based channel estimation, as shown in Figure 2, OFDM channel estimation symbols are transmitted 
periodically, and all subcarriers are used as pilots. The task here is to estimate the channel conditions (specified by $\bar{H}$ or $\bar{g}$ ) given the pilot signals (specified by matrix by $\underline{X}$ or vector $\bar{X}$ ) and received signals (specified by $\bar{Y}$ ), with or without using certain knowledge of the channel statistics. The receiver uses the estimated channel conditions to decode the received data inside the block until the next pilot symbol arrives. The estimation can be based on least square (LS), minimum meansquare error (MMSE), and modified MMSE.

\subsection{LS Estimator}

The LS estimator minimizes the parameter $(\bar{Y}-\underline{X} \bar{H})^{H}(\bar{Y}-$ $\underline{X} \bar{H})$, where $(\ldots)^{H}$ means the conjugate transpose operation. It is shown that the LS estimator of $\bar{H}$ is given by [2].

$$
\begin{aligned}
\widehat{\mathrm{H}}_{\mathrm{LS}} & =\underline{X}^{-1} \bar{H} \\
& =\left[\left(X_{k} / Y_{k}\right)\right]^{T} \quad(\mathrm{k}=0,1 \ldots . . \mathrm{N}-1)
\end{aligned}
$$

Without using any knowledge of the statistics of the channels, the LS estimators are calculated with very low complexity, but they suffer from a high mean-square error.

\subsection{MMSE Estimator}

The MMSE estimator employs the second-order statistics of the channel conditions to minimize the mean-square error.

Denote by $\underline{R}_{g g}, \underline{R}_{H H}$, and $\underline{R}_{Y Y}$ the auto-covariance matrix of $\bar{g}, \bar{H}$, and $\bar{Y}$, respectively, and by $\underline{R}_{g Y}$, the cross covariance matrix between $\bar{g}$ and $\bar{H}$. Also denote by $\sigma_{N}^{2}$ the noise variance $\mathrm{E}\left\{|\bar{N}|^{2}\right\}$. Assume the channel vector $\bar{g}$ and the noise $\bar{N}$ are uncorrelated, it is derived that

$$
\begin{aligned}
& \underline{R}_{H H}=\mathrm{E}\left\{|\bar{H} \bar{H}|^{\mathrm{H}}\right\}=\mathrm{E}\left\{(\underline{F} \bar{g})(\underline{F} \bar{g})^{\mathrm{H}}\right\}=\underline{F} \underline{R}_{g g} \underline{\mathrm{F}}^{\mathrm{H}} \\
& \underline{R}_{g Y}=\mathrm{E}\left\{|\bar{g} \bar{Y}|^{\mathrm{H}}\right\}=\mathrm{E}\left\{\bar{g}(\underline{X F} \bar{g}+\bar{N})^{\mathrm{H}}\right\}=\underline{R}_{g g} \underline{\mathrm{F}}^{\mathrm{H}} \underline{X}^{\mathrm{H}} \\
& \underline{R}_{Y Y}=\mathrm{E}\left\{\bar{Y} \bar{Y}^{\mathrm{H}}\right\}=\underline{X F R_{g}} \underline{\mathrm{F}}^{\mathrm{H}} \underline{X}^{\mathrm{H}}+\sigma_{N}^{2} \underline{I}_{N}
\end{aligned}
$$

Assume $\underline{R}_{g g}$, (thus $\underline{R}_{H H}$ ) and $\sigma_{N}^{2}$ are known at the receiver in advance, the MMSE estimator of $\bar{g}$ is given by $\widehat{\mathrm{g}}_{\mathrm{MMSE}}=$ $\underline{R}_{g Y} \underline{R}_{Y Y} \bar{Y}^{H H}$ [2-5]. Note that if $\bar{g}$ is not Gaussian $\widehat{\mathrm{g}}_{\mathrm{MMSE}}$, is not necessarily a minimum mean-square error estimator, but it is still the best linear estimator in the mean-square error sense. At last, it is calculated that

$$
\begin{aligned}
\widehat{\mathrm{H}}_{\mathrm{MMSE}} & =\underline{F} \widehat{\mathrm{g}}_{\mathrm{MMSE}}=\underline{F}\left[\left(\underline{F}^{H} \underline{X}^{H}\right)^{-1} \underline{R}_{g g}{ }^{-1} \sigma_{N}^{2}+X F\right]^{-1} \bar{Y} \\
& =\underline{F R}_{g g}\left[\left(\underline{F}^{H} \underline{X}^{H} \underline{X F}\right)^{-1} \sigma_{N}^{2}+\underline{R}_{g g}\right] \underline{F}^{-1} \widehat{\mathrm{H}}_{\mathrm{LS}}
\end{aligned}
$$

The MMSE estimator yields much better performance than LS estimators, especially under the low SNR scenarios. A major drawback of the MMSE estimator is its high computational complexity, especially if matrix inversions are needed each time the data in $\underline{X}$ changes.

\section{SIMULATION RESULTS}

In general, the 2D channel estimation schemes outperform the 1D schemes by exploiting the 2D correlations at the expense of higher computational complexity and larger time delay. Also, the block-type pilot channel estimation schemes are more suitable for the slow fading channels, and the comb-type pilot channel estimation schemes are more suitable for the middle and fast fading channels. In addition, block-type pilot schemes are used over middle or fasting fading channels, the channel estimation error may vary considerably as a function of the location of the data blocks with respect to the pilot block. The result may be a periodic variation of the decoding error rates for different OFDM blocks.

Table I show Bit Error Rate (BER) Vs Signal to noise ratio(SNR) while apply no channel estimation \& by applying least square (LS), minimum mean-square error (MMSE) as table clearly indicated that LS method will produce better signal o noise ratio by considering $\mathrm{BW}=8.75 \mathrm{Mhz}$, FFT Size $=1024, \mathrm{~T}_{\mathrm{s}}=1 \mathrm{e}-7$ where as $\mathrm{CP}$ is fixed at $1 / 8$

Table I: BER Vs SNR for diff Channel Estimation Techniques

\begin{tabular}{|l|c|c|c|c|}
\hline \multirow{2}{*}{$\begin{array}{l}\text { Sr. } \\
\text { No }\end{array}$} & \multirow{2}{*}{ SNR } & \multicolumn{3}{|c|}{ BER } \\
\cline { 2 - 5 } & & $\begin{array}{c}\text { Without } \\
\text { Channel } \\
\text { Estimation }\end{array}$ & LMMSE & LS \\
\hline 1. & $\mathbf{1}$ & 0.2300 & 0.2040 & 0.1965 \\
\hline 2. & $\mathbf{2}$ & 0.2266 & 0.1927 & 0.1834 \\
\hline 3. & $\mathbf{3}$ & 0.2221 & 0.1850 & 0.1672 \\
\hline 4. & $\mathbf{4}$ & 0.2134 & 0.1770 & 0.1582 \\
\hline 5. & $\mathbf{5}$ & 0.2096 & 0.1715 & 0.1421 \\
\hline 6. & $\mathbf{6}$ & 0.2093 & 0.1667 & 0.1347 \\
\hline 7. & $\mathbf{7}$ & 0.2008 & 0.1578 & 0.1296 \\
\hline 8. & $\mathbf{8}$ & 0.1991 & 0.1568 & 0.1174 \\
\hline 9. & $\mathbf{9}$ & 0.1994 & 0.1521 & 0.1115 \\
\hline 10. & $\mathbf{1 0}$ & 0.1980 & 0.1493 & 0.1102 \\
\hline 11. & $\mathbf{1 1}$ & 0.1971 & 0.1487 & 0.1053 \\
\hline 12. & $\mathbf{1 2}$ & 0.1931 & 0.1466 & 0.1039 \\
\hline 13. & $\mathbf{1 3}$ & 0.1944 & 0.1484 & 0.1036 \\
\hline 14. & $\mathbf{1 4}$ & 0.1952 & 0.1463 & 0.1007 \\
\hline 15. & $\mathbf{1 5}$ & 0.1964 & 0.1442 & 0.0994 \\
\hline 16. & $\mathbf{1 6}$ & 0.1968 & 0.1445 & 0.1000 \\
\hline
\end{tabular}




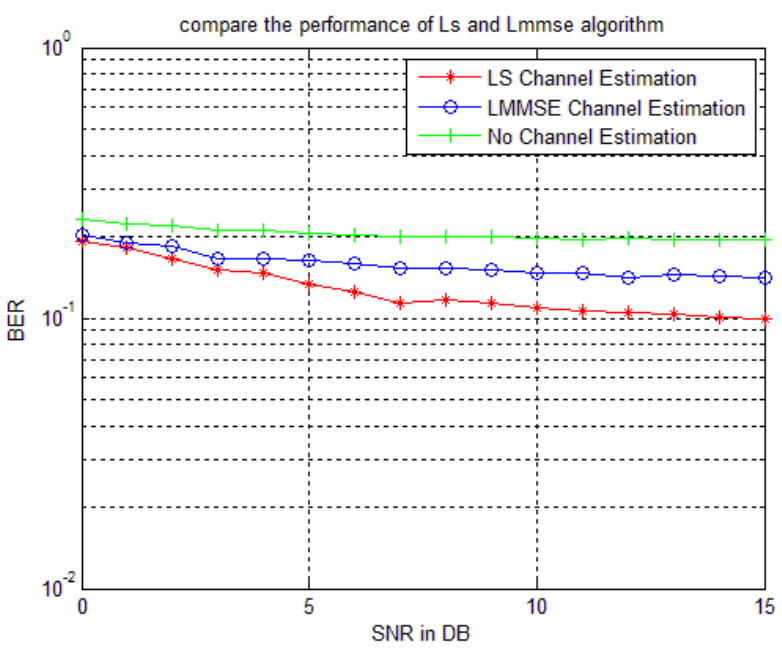

Fig 3: SNR (db) Vs BER performance of diff channel estimation (LS, LMSSE \& No Channel)

Figure 3 show performance of different block type channel estimation methods i.e. LS \& LMMSE

\section{CONCLUSION \& FUTURE SCOPE}

In this study a low complexity structure of receiver was proposed so that the LS method and linear interpolation were used for initial channel estimation. For improving accuracy of channel estimation, LMS iterative algorithm was added to receiver which includes a feedback of output and improves the BER performance of system, closed to the ideal channel performance.

For future research, the method to determine the pilots location, to improve efficiency of the system may be considered.

\section{REFERENCES}

[1] Edfors, O., Sandell, M., Van de Beek, J.-J., Landström, D., and Sjöberg, F., An Introduction to Orthogonal Frequency Division Multiplexing, Luleå, Sweden: Luleå Tekniska Universitet, 1996, pp. 1-58.

[2] Van de Beek, J.-J., Edfors, O. S., Sandell, M., Wilson, S. K., and Börjesson, O. P., "On channel estimation in OFDM systems," 45th IEEE Vehicular Technology Conference, Chicago, Il., vol. 2, pp. 815-819, July 1995.
[3] Edfors, O., Sandell, M., Van de Beek, J.-J., and Wilson, S. K., "OFDM Channel Estimation by Singular Value Decomposition," IEEE Transactions on Communications, vol. 46, pp. 931-939, July 1998.

[4] Strobach, P., "Low-Rank Adaptive Filters," IEEE Transactions on Signal Processing, vol. 44, pp. 2932 2947. Dec. 1996.

[5] Coleri, S., Ergen, M., Puri, A., and Bahai, A., "Channel Estimation Techniques Based on Pilot Arrangement in OFDM Systems, " IEEE Transactions on Broadcasting, vol. 48, pp. 223-229, Sept. 2002.

[6] $\mathrm{Wu}$, J., and $\mathrm{Wu}, \mathrm{W} .$, "A Comparative Study of Robust Channel Estimators for OFDM Systems," Proceedings of ICCT, pp. 1932-1935, 2003.

[7] Yang, B., Letaief, K. B., Cheng, R. S., and Cao, Z., "Channel Estimation for OFDM Transmission in Multipath Fading channels Based on Parametric Channel Modeling," IEEE Transactions on Communications, vol. 49, pp. 467-479, March 2001.

[8] Ottersten, B., Viberg, M., and Kailath, T., "Performance Analysis of the Total Least Squares ESPRIT Algorithm," IEEE Transactions on Signal Processing, vol. 39, pp. 1122-1135, May 1991

[9] Hou, X., Li, S., Liu, D., Yin, C., and Yue, G., "On Twodimensional Adaptive Channel Estimation in OFDM Systems," 60th I9EEE Vehicular Technology Conference, Los Angeles, Ca., vol. 1, pp. 498-502, Sept. 2004.

[10] Sanzi, F., Sven, J., and Speidel, J., "A Comparative Study of Iterative Channel Estimators for Mobile OFDM Systems," IEEE Transactions on Wireless Communications, vol.2, pp. 849-859, Sept. 2003.

[11] Li, Y., "Simplified Channel Estimation for OFDM Systems with Multiple Transmit Antennas," IEEE Transactions on Communications, vol. 1, pp. 67-75, January 2002.

[12] Auer, G., "Channel Estimation in Two Dimensions for OFDM Systems with Multiple Transmit Antennas," GLOBECOM, pp. 322-326, 2003.

[13] IEEE P802.16 (Draft 8, May 2005), WiMAX Specification. 